\title{
Convex optimization of virtual storage system scheduling in market environment
}

\author{
Peng HOU ${ }^{1}$, Junjie HU $^{2}$ ㅁ, Guangya YANG ${ }^{1}$
}

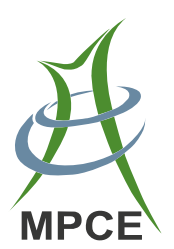

\begin{abstract}
Due to the popularization of distributed energy resources (DERs), the aggregated prosumer effect excels a general energy storage system characteristic. Virtual energy storage system (VESS) concept is proposed hereby that mimics an actual storage unit and incorporates the same charging (consumer) and discharging (producer) modes. It is possible to provide ancillary services via VESS by exploiting the flexibility and thus much research has been proposed on the optimization of the VESS scheduling. In general, the charging and discharging efficiencies of VESS are different and there can be only one status at a time slot. To achieve the optimal schedule while considering the constraints above, binary terms should be introduced into the optimization problem which end up with a nonconvex problem. In this paper, a complimentary mathematical proof is given for the convexification of this mixed integer linear programming (MILP) problem so that the linear programming (LP) method can be applied instead if the objective function is linear. The proposed proof is
\end{abstract}

CrossCheck date: 26 March 2019

Received: 27 September 2018/ Accepted: 26 March 2019/Published online: 25 July 2019

(C) The Author(s) 2019

$\triangle$ Junjie HU

junjiehu@ncepu.edu.cn

Peng HOU

pehou@elektro.dtu.dk

Guangya YANG

gyy@elektro.dtu.dk

1 Center of Electrical Engineering, Technical University of Denmark, Elektrovej, Building 325, Lyngby, Denmark

2 State Key Laboratory of Alternate Electrical Power System with Renewable Energy Sources, North China Electric Power University, Beijing 102206, China validated through a case study and the simulation results show the effectiveness of the proposed method.

Keywords Virtual energy storage system (VESS), Convexification, Mixed integer linear programming (MILP), Complimentary mathematical proof

\section{Introduction}

Due to the increasing penetration of DERs such as photovoltaic (PV), electric vehicle (EV), and battery storage devices, the problems of congestion and voltage violation are expected to appear which challenges the safe operation of the power system [1]. Demand response is one of the enabling solutions for solving such a problem and thus draws attention worldwide [2]. To facilitate an effective demand response, the individual consumption and production could be aggregated as a VESS and controlled by the aggregator. In [3], a method for solving optimal power flow in a hybrid PV, wind and storage power system was proposed, the charging/discharging schedule of the battery was obtained by a linear programming (LP) model since the charging and discharging efficiencies were assumed to be the same. The same LP model was also adopted in [4] to get the optimal schedule of energy storage in a network of grid-connected micro-grids. Considering the vehicle to grid (V2G) function, the scheduling problem of VESS is similar to EV, where its charging and discharging schedule considering the varying electricity prices in the different market is optimized. Considering the reserve margin commitment when participation in the day ahead market (DAM), the EV's schedule was optimized by a two-stage stochastic MILP model in [5]. Taken the detailed battery wear cost model into account when 
providing V2G service, the EV's scheduling was also formulated as a MILP problem in [6]. Similar to the EV's optimization problem, a MILP based rolling optimization work was presented in [7] for optimizing the VESS for a smart home with PV and battery.

It can be seen that the scheduling problem of VESS was usually solved with MILP model [5-7] which is timeconsuming. However, the precondition of using the LP model requires the bi-directional efficiency must be the same, which is not the real case. To convexify the problem, a proof in [8] was given whereas we found it is only a necessary but not sufficient condition which may incur misunderstanding. Thus, a complimentary proof for [8] is given in this paper to give a general condition of using the LP model.

\section{Problem formulation}

Considering the varying electricity price in DAM, the schedule of VESS at a particular hour can be optimized with the following model [6].

$$
\begin{array}{ll}
\min \left(P_{t}^{+}+P_{t}^{-}\right) \lambda_{t}^{D A} \\
\text { s.t. } \quad \delta_{t}^{a}, \delta_{t}^{b} \in\{0,1\} \\
& \delta_{t}^{a}+\delta_{t}^{b} \leq 1 \\
& 0 \leq P_{t}^{+} \leq \delta_{t}^{a} P_{\max , \mathrm{ch}} \\
& -\delta_{t}^{b} P_{\max , \mathrm{dis}} \leq P_{t}^{-} \leq 0 \\
& S O C_{t+1}=S O C_{t}+\left(P_{t}^{+} \eta_{+}+P_{t}^{-} \eta_{-}^{-1}\right) E_{b}^{-1} \\
& S O C_{1}=S O C_{\text {init }} \\
& S O C_{\text {end }}=S O C_{\text {des }} \\
& S O C_{\min } \leq S O C_{t} \leq S O C_{\max }
\end{array}
$$

where $\lambda^{D A}$ is the predicted DAM price, Dkk (Danish Krone); $t$ is the time index; $\delta^{a}, \delta^{b}$ are the charging and discharging mode indicators; $\eta_{+}, \eta_{-}$are the charging and discharging efficiency; $E_{b}$ is the capacity of VESS, $\mathrm{kWh}$; $P^{+}, P^{-}$are the charging and discharging schedules of VESS, kW; $P_{\text {max,ch }}, P_{\text {max,dis }}$ are the maximum charging and discharging power limits of VESS, $\mathrm{kW}$; $S O C_{\text {init }}, S O C_{\mathrm{min}}$, $S O C_{\max }, S O C_{d e s}, S O C_{\text {end }}$ are the initial state of charge (SOC), minimum, maximum SOC, desired final SOC, and SOC at the end of the day, respectively.

It can be seen that the above problem is formulated as a MILP model and thus can be solved by the existing solver such as Gurobi [9].

\section{Mathematical proof}

To convexify the above problem, a proof is given by [8] so that the LP method can be applied instead assuming a linear objective function. However, we find that the method does not always work and thus would like to provide a complimentary proof and simulation validation on the conditions when the assumption will work and when will not on principle.

Proof I Assume $P_{t}^{+} \geq 0$ and $P_{t}^{-} \leq 0$ are the optimal charging and discharging solution satisfying $P_{t}^{+} P_{t}^{-} \neq 0$, for any $t \in[1, N] . N$ is the total number of time slots. Let $Q_{t}^{+}=$ $P_{t}^{+}-\varepsilon$ and $Q_{t}^{-}=P_{t}^{-}+\varepsilon \eta_{+} \eta_{-}$to be another solution while $\varepsilon$ represents a small positive value. Those two solutions yield the same change of SOC as:

$Q_{t}^{+} \eta_{+}+\frac{Q_{t}^{-}}{\eta_{-}}=P_{t}^{+} \eta_{+}+\frac{P_{t}^{-}}{\eta_{-}}$

Under one price scheme, considering the objective is to minimize charging cost or maximize discharging benefit, thus one obtains:

$\left(Q_{t}^{+}+Q_{t}^{-}\right) \lambda_{t}=\left(P_{t}^{+}+P_{t}^{-}\right) \lambda_{t}-\varepsilon\left(1-\eta_{+} \eta_{-}\right) \lambda_{t}$

The optimality does not stand if the following condition is met:

$\left(Q_{t}^{+}+Q_{t}^{-}\right) \lambda_{t}<\left(P_{t}^{+}+P_{t}^{-}\right) \lambda_{t}$

where $\lambda_{t}$ is the electricity price at time slot $t$. Equation (12) means a lower cost is realized by the new solution. In other words, the assumed solution is not optimal. By taking (11) into (12), to keep the optimality, we must have

$0<\varepsilon\left(1-\eta_{+} \eta_{-}\right) \lambda_{t}$

From (13), the conditions for solving the optimization problem using LP method can be concluded in Table 1.

Similarly, it can also be proved that the sign of $P_{t}^{+}$and $P_{t}^{-}$does not influence the conclusion in Table 1. Taking $P_{t}^{+} \geq 0$ and $P_{t}^{-} \geq 0$ as an example and following the similar

Table 1 Optimality condition

\begin{tabular}{ll}
\hline Condition 1 & Condition 2 \\
\hline$\lambda_{t}>0$ & $\lambda_{t}<0$ \\
$\eta_{+} \eta_{-}<1$ & $\eta_{+} \eta_{-}>1$ \\
Realistic & Unrealistic \\
\hline
\end{tabular}


procedure in Proof I, let $Q_{t}^{+}=P_{t}^{+}-\varepsilon$ and $Q_{t}^{-}=P_{t}^{-}-\varepsilon \eta_{+} \eta_{-}$ to be another solution, then

$Q_{t}^{+} \eta_{+}-\frac{Q_{t}^{-}}{\eta_{-}}=P_{t}^{+} \eta_{+}-\frac{P_{t}^{-}}{\eta_{-}}$

The optimality does not stand if the following condition is met:

$\left(Q_{t}^{+}-Q_{t}^{-}\right) \lambda_{t}<\left(P_{t}^{+}-P_{t}^{-}\right) \lambda_{t}$

At last, the final condition can also be concluded as (13).

Besides, it is known that there can be two prices for the same time slot in balancing electricity market as the up/down regulation price. To enable the LP method in such an application, another proof is given in the following.

Proof II Following the same assumption in Proof I, it is assumed that in this case, the two prices $\left(\lambda_{1, t}, \lambda_{2, t}\right)$ are above zero, then the objective of (11) can be modified as follow:

$Q_{t}^{+} \lambda_{1, t}+Q_{t}^{-} \lambda_{2, t}=\left(P_{t}^{+}-\varepsilon\right) \lambda_{1, t}+\left(P_{t}^{-}+\varepsilon \eta_{+} \eta_{-}\right) \lambda_{2, t}$

Similarly, $\quad$ if $\quad Q_{t}^{+} \lambda_{1, t}+Q_{t}^{-} \lambda_{2, t}<P_{t}^{+} \lambda_{1, t}+P_{t}^{-} \lambda_{2, t}$, the assumption does not stand. By taking (16) into $Q_{t}^{+} \lambda_{1, t}+Q_{t}^{-} \lambda_{2, t}<P_{t}^{+} \lambda_{1, t}+P_{t}^{-} \lambda_{2, t}$, we can get:

$-\varepsilon\left(\lambda_{1, t}-\eta_{+} \eta_{-} \lambda_{2, t}\right)<0 \Rightarrow \frac{\lambda_{1, t}}{\lambda_{2, t}}>\eta_{+} \eta_{-}$

It can be seen that another condition (17) should be added on top of the conditions concluded in Table 1 for solving a two-price optimization problem using LP.

\section{Results and discussion}

In this part, the optimization of the EV owner's schedule considering $\mathrm{V} 2 \mathrm{G}$ is selected as the case study. The parameters of EV are listed in Table 2 while the electricity price for each scenario is shown in Fig. 1. In Fig. 1a, blue curve represents the one price scheme. In Fig. $1 b$, blue curve denotes the value of $\lambda_{2, t}$ and red curves means the value of $\lambda_{1, t}$.

To demonstrate the conclusion from our proof, the convexification method in [8] is compared with a MILP model in each scenario.

Table 2 EV battery parameters

\begin{tabular}{llllll}
\hline $\begin{array}{l}\text { Battery size } \\
(\mathrm{kWh})\end{array}$ & $\begin{array}{l}S O C_{\min } \\
(\%)\end{array}$ & $\begin{array}{l}S O C_{\max } \\
(\%)\end{array}$ & $\begin{array}{l}\text { Power } \\
(\mathrm{kW})\end{array}$ & $\begin{array}{l}\eta_{+} \\
(\%)\end{array}$ & $\begin{array}{l}\eta_{-} \\
(\%)\end{array}$ \\
\hline 25 & 20 & 85 & 5.28 & 90 & 95 \\
\hline
\end{tabular}

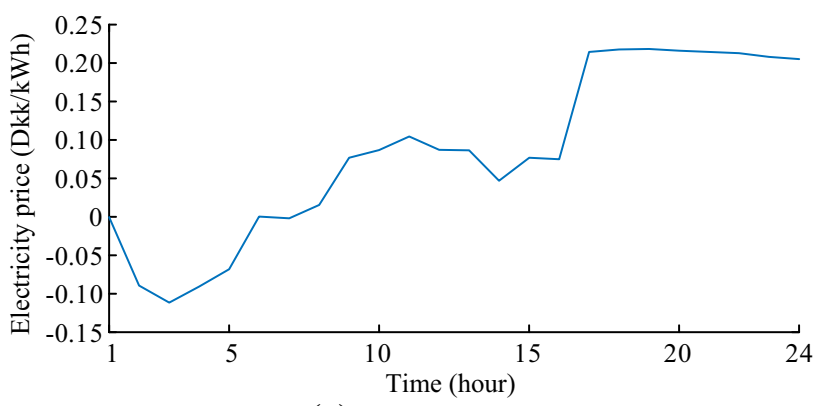

(a) Prices for scenario I

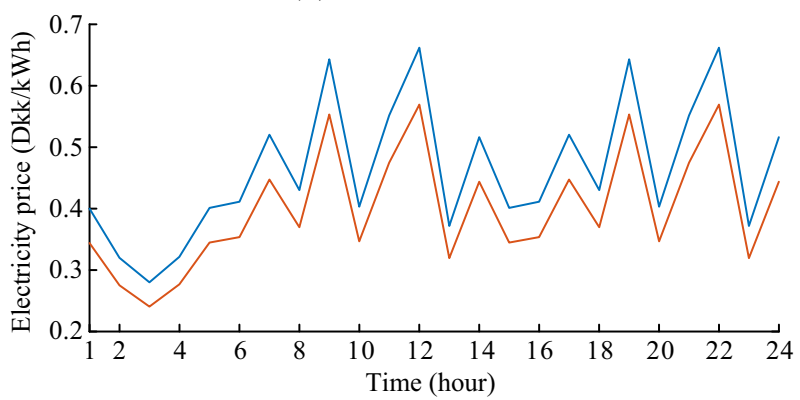

(b) Prices for scenario II

Fig. 1 Electricity price for different scenario

\subsection{Scenario I: EV optimal schedule with $\eta_{+} \eta_{-}<1$}

In this scenario, the optimal schedule is obtained based on the prices illustrated in Fig. 1a. The final solution is shown in Fig. 2.

It can be seen in Fig. 2 that the charging and discharging status still exist simultaneously at some time (hours 5, 8 and 21) though $\eta_{+} \eta_{-}<1$ when LP model is adopted, this phenomenon contracts with the conclusion in [8]. Note that the blue bar represents charging power, and the red bar

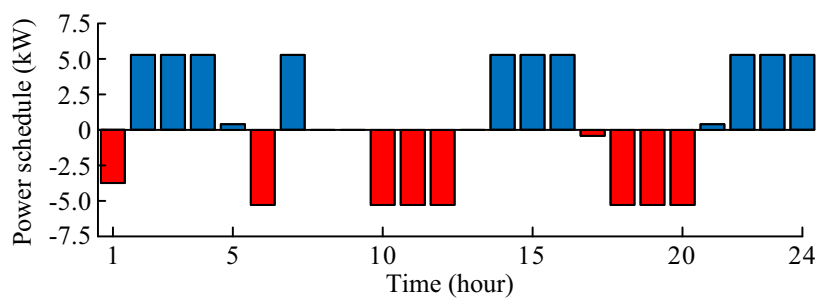

(a) MILP method

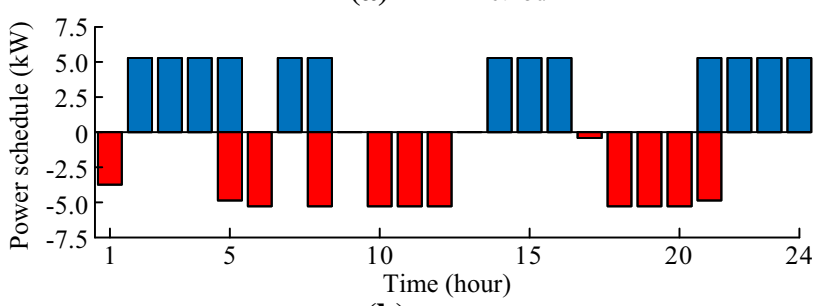

(b) LP method

Fig. 2 Power schedule comparison for two methods (Scenario I) 


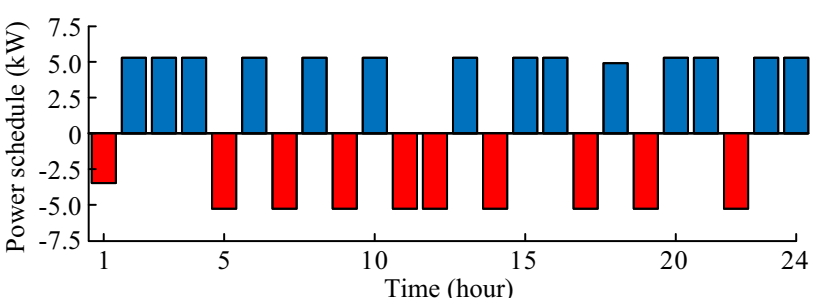

(a) MILP method

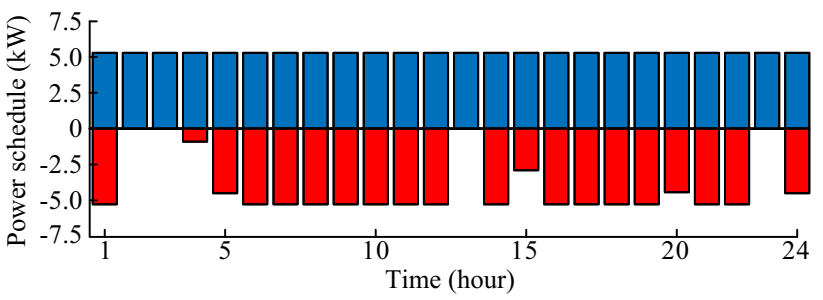

(b) LP method

Fig. 3 Power schedule comparison for two methods with $\lambda_{1, t}$ $\lambda_{2, t}=0.84$

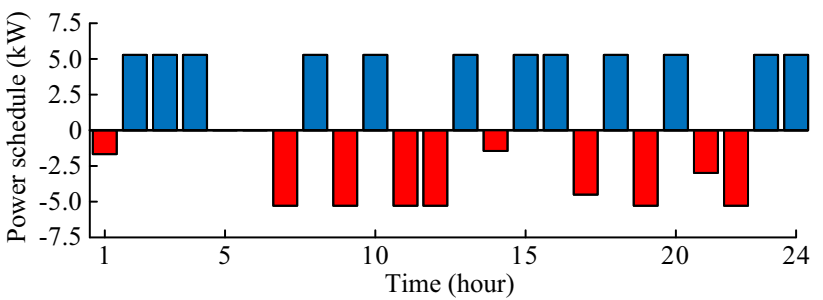

(a) MILP method

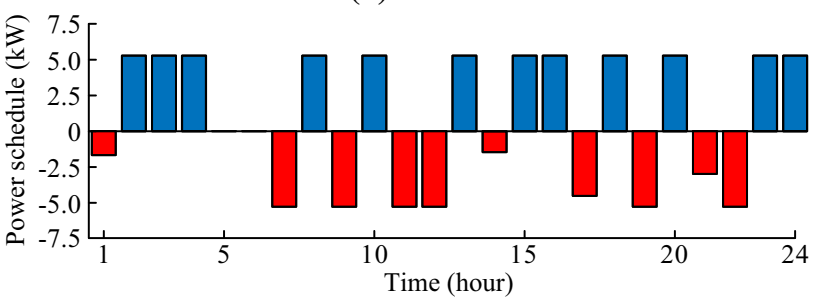

(b) LP method

Fig. 4 Power schedule comparison for two methods $\lambda_{1, t} / \lambda_{2, t}=0.86$

denotes discharging power. These signs also apply to Fig. 3 and Fig. 4.

\subsection{Scenario II: EV optimal schedule with $\eta_{+} \eta_{-}<1$ and different ratio of $\lambda_{1, t} / \lambda_{2, t}$}

Based on proof I and II, it can be known that (17) should be met so that LP model can be adopted instead, and thus we design a time series price with a ratio of $\lambda_{1, t} / \lambda_{2, t}$ equals to 0.84 and 0.86 , respectively $\left(\eta_{+} \eta_{\text {- }}\right.$ equals to 0.855$)$. The final solution using MILP or LP model with ratio equals to 0.84 and 0.86 are illustrated in Figs. 3 and 4, respectively.
Compared Fig. 3 to Fig. 4, it can be seen that LP method is effective when $\lambda_{1, t} / \lambda_{2, t}=0.86$ while fails when $\lambda_{1, t} / \lambda_{2, t}=0.84$ which correspond to the Proof II.

\section{Conclusion}

From our proof, we can see that the conditions of applying the LP method for solving the EV optimal schedule problem can be listed as follows:

1) The product of charging and discharging efficiency should be lower than $1\left(\eta_{+} \eta_{-}<1\right)$.

2) The time series electricity price should be above zero.

3) If there are two price indicators to be considered in the optimization problem, for instance, the stochastic optimization problem, extra condition as (17) should also be satisfied so that the LP method can be applied.

4) The signs of optimization variables do not influence the effectiveness of the convexification method.

Acknowledgements This work was supported by Energy Technology Development and Demonstration Program (No. EUDP171:(12551)) and National Natural Science Foundation of China (No. 51877078).

Open Access This article is distributed under the terms of the Creative Commons Attribution 4.0 International License (http:// creativecommons.org/licenses/by/4.0/), which permits unrestricted use, distribution, and reproduction in any medium, provided you give appropriate credit to the original author(s) and the source, provide a link to the Creative Commons license, and indicate if changes were made.

\section{References}

[1] Reddy SS, Bijwe PR (2016) Day-ahead and real time optimal power flow considering renewable energy resources. Electr Power Energy Syst 82:400-408

[2] Reddy SS, Abhyankar AR, Bijwe PR (2015) Joint market clearing of energy and demand response offers considering voltage dependent load models. J Electr Syst 11-4:433-446

[3] Reddy SS (2017) Optimal power flow with renewable energy resources including storage. Electr Eng 99:685-695

[4] Wang D, Meng K, Gao X et al (2018) Coordinated dispatch of virtual energy storage systems in LV grids for voltage regulation. IEEE Trans Ind Informat 14(6):2452-2462

[5] Sánchez-Martín P, Lumbreras S, Alberdi-Alén A (2016) Stochastic programming applied to EV charging points for energy and reserve service markets. IEEE Trans Power Syst 31(1):198-205

[6] Farzin H, Fotuhi-Firuzabad M, Moeini-Aghtaie M (2016) A practical scheme to involve degradation cost of Lithium-ion batteries in vehicle-to-grid applications. IEEE Trans Sustain Energy 7(4):1730-1738 
[7] Wang H, Meng K, Dong ZY et al (2015) Efficient real-time residential energy management through MILP based rolling horizon optimization. In: Proceedings of 2015 IEEE PES general meeting, Denver, USA, 26-30 July 2015, pp 1-6

[8] Hao H, Wu D, Lian J et al (2018) Optimal coordination of building loads and energy storage for power grid and end user services. IEEE Trans Smart Grid 9(5):4335-4345

[9] "Gurobi optimizer". http://www.gurobi.com/. Accessed 27 September 2018

Peng HOU received his B.E. degree in Hebei University of Technology, Tianjin, China, in 2008. He received his master degree in Chalmers University of Technology, Gothenburg, Sweden, in 2010 and Ph.D. degree at the Energy Department in Aalborg University, Denmark, in 2017. He is currently a postdoc researcher at the Technical University of Denmark, Lyngby, Denmark. His research interests include operational research, mathematical programming, heuristic optimization, wind energy, electrolysis storage system, electricity market analysis and optimization.

Junjie HU received his M.Sc. degree in control theory and control engineering from Tongji University, Shanghai, China, in 2010, and the Ph.D. degree in electrical engineering from the Technical University of Denmark, Denmark, in 2014. He was a postdoc researcher with the Department of Electrical Engineering, Technical University of Denmark. He is currently an Associate Professor with School of Electrical and Electronic Engineering, North China Electric Power University, China. His research interests include distributed energy resources energy management and their participations in frequency ancillary service markets.

Guangya YANG received the B.E., M.E., and Ph.D. degrees all in the field of the electric power system, in 2002, 2005, and 2008, respectively. Since 2009 , he has been with the Technical University of Denmark, Kongens Lyngby, Denmark, as a Postdoctoral Researcher, and he is currently an Associate Professor with the Center for Electric Power and Energy, Department of Electrical Engineering, Technical University of Denmark. Since 2009, he has been leading several industrial collaborative projects in Denmark in the field of monitoring, operation, and protection of renewable energy systems. His research interests include renewable energy integration, smart grids, and cyber-physical energy systems. 